\title{
Bulk Crystal Growth, and High-Resolution X-Ray Diffraction Results of LiZnP Semiconductor Material
}

\author{
Benjamin W. Montag ${ }^{1}$, Michael A. Reichenberger ${ }^{1}$, Madhana Sunder ${ }^{2}$, Philip B. Ugorowski ${ }^{1}$, \\ Kyle A. Nelson ${ }^{1}$, Douglas S. McGregor ${ }^{1}$ \\ ${ }^{1}$ Semiconductor Materials and Radiological Technologies (S.M.A.R.T) Laboratory, Kansas State \\ University, Manhattan, KS 66506, U.S.A. \\ ${ }^{2}$ Bruker AXS Inc, 5465 E. Cheryl Parkway, Madison, WI 53711, U.S.A.
}

\section{Corresponding Author Contact:}

Benjamin W. Montag

Email: bmontag@ksu.edu

Tel: 785:532:6480

Fax: 785:532:7057

Keywords: B1. Lithium compounds; A1. Radiation; B2 Semiconducting ternary compounds; A1. X-ray diffraction; A1. Crystal Structure; A2. Bridgman technique

\section{ABSTRACT}

Nowotny-Juza compounds continue to be explored as a candidate for solid-state neutron detectors. Such a device would have greater efficiency, in a compact form, than present day gasfilled ${ }^{3} \mathrm{He}$ and ${ }^{10} \mathrm{BF}_{3}$ detectors. The ${ }^{6} \mathrm{Li}(\mathrm{n}, \mathrm{t}){ }^{4} \mathrm{He}$ reaction yields a total $\mathrm{Q}$ value of $4.78 \mathrm{MeV}$, larger than ${ }^{10} \mathrm{~B}$, an energy easily identified above background radiations. Hence, devices fabricated from semiconducting compounds containing either natural $\mathrm{Li}$ (nominally $7.5 \%{ }^{6} \mathrm{Li}$ ) or enriched ${ }^{6} \mathrm{Li}$ (usually 95\% ${ }^{6} \mathrm{Li}$ ) may provide a semiconductor material for compact high efficiency neutron detectors. Starting material was synthesized by preparing equimolar portions of $\mathrm{Li}, \mathrm{Zn}$, and $\mathrm{P}$ sealed under vacuum $\left(10^{-6} \mathrm{Torr}\right)$ in quartz ampoules lined with boron nitride and subsequently reacted in a compounding furnace [1]. The synthesized material showed signs of high impurity levels from material and electrical property characterizations. A static vacuum sublimation in quartz was performed to help purify the synthesized material [2]. Bulk crystalline samples were grown from the purified material. An ingot $9.6 \mathrm{~mm}$ in diameter and $4.0 \mathrm{~mm}$ in length was harvested. Individual samples were characterized for crystallinity on a Bruker AXS Inc. D2 CRYSO, energy dispersive x-ray diffractometer, and a Bruker AXS D8 DISCOVER, high-resolution $\mathrm{x}$-ray diffractometer with a $0.004^{\circ}$ beam divergence. The (220) orientation was characterized as the main orientation with the D2 CRYSO, and confirmed with the D8 DISCOVER. An out-of-plane high-resolution rocking curve yielded a $0.417^{\circ}$ full width at half maximum (FWHM) for the (220) LiZnP. In-plane ordering was confirmed by observation of the (311) orientation, where a rocking curve was collected with a FWHM of $0.294^{\circ}$.

\section{INTRODUCTION}

Nowotny-Juza compounds were originally, and are still today, studied for photonic applications [3-5]. The filled tetrahedral compound class $\mathrm{A}^{\mathrm{I}} \mathrm{B}^{\mathrm{II}} \mathrm{C}^{\mathrm{V}}$ materials consist of the III-Vlike compounds with lithium interstitials. These materials are desirable for their zincblende cubic 
crystal structure, and unlike thin-film and doped devices, the concentration of $\mathrm{Li}$ atoms is equal to other constituent atoms, as seen in Fig. 1. The zincblende crystal structure is arranged where the group II atom is located at $\tau_{1}=(0,0,0) a$ where $a$ is the lattice constant, and the group V atom is located at $\tau_{2}=(1 / 4,1 / 4,1 / 4) a$. The spacious cubic structure allows for lithium atoms to fill the interstitial site at $\tau_{3}=(1 / 2,1 / 2,1 / 2) a$ as shown in Fig. $1[6,7]$. The filling of these interstitial sites allows for a lithium-loaded semiconducting material. A variety of the ternary compounds have been synthesized in the past, commonly in graphite lined quartz ampoules [8]. Synthesis in a tantalum lining crucible has also been reported [6]. However, bulk growth of these materials has not been reported. The existing material and electrical properties have been reported from samples procured by direct reaction of elemental and/or binary materials. These samples are generally tiny facets extracted from the reaction mixture, and are usually small and extremely difficult to handle and process into devices

Materials containing ${ }^{6} \mathrm{Li},{ }^{10} \mathrm{~B},{ }^{113} \mathrm{Cd},{ }^{157} \mathrm{Gd}$ and ${ }^{199} \mathrm{Hg}$ have been considered for solid-state neutron detectors [9-20]. The ${ }^{10} \mathrm{~B}(\mathrm{n}, \alpha)^{7} \mathrm{Li}$ reaction is desirable for the ${ }^{10} \mathrm{~B}$ microscopic thermal neutron absorption cross section of 3839 barns, but boron-based compounds, such as BP, BN, and BAs have shown limited success, and thus far do not appear promising due to crystal growth and materials preparation problems [17-20]. Thin-film boron devices suffer due to their geometry, where only one reaction product can be absorbed in semiconducting material, therefore producing some signals that can be difficult to distinguish from background and gamma-ray induced events [21]. Additionally, because of the reaction product self-absorption, the reactive film thickness is restricted, and therefore thermal neutron absorption is limited, consequently resulting in a maximum intrinsic detection efficiency of approximately $4.5 \%$ [21]. Solid-state detectors containing ${ }^{113} \mathrm{Cd}$ and ${ }^{199} \mathrm{Hg}$ devices also have limited detection efficiency due to the low absorption probability of the prompt gamma-rays that result from ${ }^{113} \mathrm{Cd}(\mathrm{n}, \gamma){ }^{114} \mathrm{Cd}$ and ${ }^{199} \mathrm{Hg}(\mathrm{n}, \gamma){ }^{200} \mathrm{Hg}$ reactions [9-12]. The reaction ${ }^{157} \mathrm{Gd}(\mathrm{n}, \gamma){ }^{158} \mathrm{Gd}$ is desirable for the large ${ }^{157} \mathrm{Gd}$ thermal neutron capture cross section of 240,000 barns. Unfortunately, the ${ }^{157} \mathrm{Gd}(\mathrm{n}, \gamma){ }^{158} \mathrm{Gd}$ reaction yields a spectrum of low energy prompt gamma rays and low energy conversion electrons, all of which are difficult to discern from background radiations [21]. Finally, ${ }^{6} \mathrm{Li}$ has not been explored to the same extent as other thermal neutron absorbers, and has an intrinsic thermal neutron absorption cross section of 940 barns. The reaction produces a total Q-value of $4.78 \mathrm{MeV}$, described be the following reaction,

$$
{ }^{6} \mathrm{Li}+{ }_{0}^{1} n \rightarrow{ }^{3} \mathrm{H}(2.73 \mathrm{MeV})+\alpha(2.05 \mathrm{MeV}) \quad \text {. }
$$

LiZnP has been reported to have a bandgap of $2.1 \mathrm{eV}$, ideal for a room-temperature semiconductor device [8]. A Hall mobility of $1-10 \mathrm{~cm}^{2} / \mathrm{V} \cdot \mathrm{s}$ was reported by Kuriyama [6]. These known electrical properties make LiZnP and other compounds in the class desirable for compact, high neutron sensitivity, solid-state detectors. Synthesis of Nowotny-Juza materials is not trivial. Lithium is very reactive, and the high-pressures that are developed due to the exothermic reactions that occur during the reaction process often result in containment failure. It was found that making the alloy, $\mathrm{Li}-\mathrm{Zn}$, prior to reaction aided the reaction process, and resulted in a much higher ampoule yield [1]. Additionally, melting temperatures of the class of materials have not been completely characterized $[6,8,22]$. Reported melting temperatures were not consistent with what was observed in the following study. Unfortunately, the difficulties in the synthesis and handling process limit the available physical and electrical property data $[3-8,22$, 23]. 


\section{EXPERIMENTAL DETAILS}

95

96

97

98

99

100

101

102

103

104

105

106

107

108

109

110

111

112

113

114

115

116

117

118

119

120

121

122

123

124

125

126

127

128

129

130

131

132

133

134

135

136

Described in the following work is a method developed for the bulk crystal growth process of LiZnP, post processing methods, and the high-resolution XRD characterization.

\section{Bulk Crystal Growth}

LiZnP was synthesized, as described elsewhere, in small batches up to 2.0 grams [1]. The synthesized material was purified by a static sublimation process. The process appears to successfully separate the ternary LiZnP material from elemental and binary residual materials that often result from the synthesis process [2]. This purified LiZnP material was subsequently grown into bulk crystals under a high-temperature vertical Bridgman technique.

A 0.5 inch diameter tantalum tube ( 0.02 inch wall thickness) was cut into a 2.9375 inch long sample containment tube. Tantalum caps were punched, and formed from 0.02 inch thick tantalum sheet. A pyrolytic graphite crucible was custom designed to fit comfortably in the tantalum tube (supplied by Mersen, Inc.). Each of the components were cleaned of oils and contaminants by ultrasonic vibration in a series of solvents namely, isopropanol, acetone and trichloroethylene. The components were loaded into an argon glove box, with minimal oxygen and moisture contamination of less than $0.1 \%$. The bottom cap was welded on the tantalum tube by a Maxstar 150 STL arc welder. The welding torch / stick was fed through the glove box, and the argon environment allowed for the arc plasma to form, and therefore allowed for welding within the box. Purified LiZnP material $(1.19 \mathrm{~g})$ was produced, as detailed elsewhere $[1,2]$ was loaded into the pyrolytic graphite crucible, which was loaded into the tantalum vessel. A tantalum cap was welded to the top of the vessel, thereby, making an air-tight containment of $\mathrm{LiZnP}$ material and atmospheric pressure argon as shown in Fig. 2.

A bulk crystal was grown in the tantalum vessel contained in a vacuum chamber, equipped with an Omega iR2C series fiber optic thermometer mounted on a two axis rotation stage for alignment purposes. The vacuum chamber was equipped with two feed-throughs connected to an R.D. Mathis LV 400 high-current, low-voltage power source. The tantalum vessel was connected between two electrical connecting oxygen-free plates, referred to as a copper harness, depicted in Fig 3. The copper harness connected to the electrical feed-throughs within a vacuum chamber and these feed-throughs connected to the high-current, low-voltage power source. Continuity was tested to confirm a stable connection outside of the vacuum chamber. The chamber was evacuated, and then purged with ultra-high purity argon at least six times. After the final purge, the chamber pressure was maintained between $300-400$ mTorr argon pressure. The current was ramped typically from 0 amps to approximately $295-315 \mathrm{amps}$ over the course of at least an hour, until the optical thermometer measured approximately $1345^{\circ} \mathrm{C}$ at the tantalum surface. Temperature was maintained for at least an hour. The system was then ramped down to room temperature over the following 40 hours. The vessel was extracted from the chamber, and opened with a pipe cutter under a pure argon environment. The ingot was harvested, and individual samples were cut from the ingot with a Laser Technology West Ltd. CS400 diamond wire saw while under a constant flow of mineral oil for protection from moisture in the air. 


\section{X-ray Diffraction Measurements}

A LiZnP sample was sliced to $4.077 \times 3.518 \times 2.020 \mathrm{~mm}$, and XRD analysis was performed on one side of the $4.077 \times 3.518 \mathrm{~mm}$ face. The sample was mounted into a polishing fixture by parafin wax. The sample face was polished with a series of silicon carbide polishing papers ranging from $15.3-2.5$ micron grain size, ANSI grit: 600 (P1200), 800 (P2400), and 1200 (P4000) [24, 25]. The polished sample was mounted under argon between two Biaxiallyoriented polyethylene terephthalate (BoPET) sheets, known as Mylar ${ }^{\circledR}$, in a custom sample holder designed to keep the sample air-tight, and then evaluated on a Bruker AXS Inc. D2 CRYSO energy-dispersive x-ray diffractometer. The measurements evaluated the crystal structure, orientation, lattice constant and $d$ spacing. The sample was then mounted into an airtight poly(methyl methacrylate) (PMMA) plastic sample holder with a beryllium dome with double sided tape as shown in Fig. 4. The particular sample was evaluated while under argon by a Bruker AXS Inc. high-resolution D8 DISCOVER x-ray diffractometer. The diffractometer was equipped with molybdenum optics with a four bounce monochromator $\left(0.004^{\circ}\right.$ beam divergence), and a line beam $12.0 \mathrm{~mm}$ tall by $9.5 \mathrm{~mm}$ width (slit size). The sample was evaluated using phase identification scans, rocking curves and off axis measurements.

\section{RESULTS AND DISSCUSSION}

\section{Bulk Crystal Growth}

The bulk crystal growth process produced ingots of various size, the largest being $13.0 \mathrm{x}$ $9.0 \times 6.0 \mathrm{~mm}^{3}$, as shown in Fig. 5. The ingot grown from purified material was $9.6 \mathrm{~mm}$ in diameter and $4.0 \mathrm{~mm}$ in length. A slice of the purified ingot is shown in Fig. 6. It was evident that the melting temperature reported by Kuriyama was not confirmed, nor observed in this study [6]. Numerous attempts to grow LiZnP by melt in crucible lined quartz ampoules always resulted in non-congruent melting up to temperatures of $1150^{\circ} \mathrm{C}$. Temperatures above $1150^{\circ} \mathrm{C}$ in quartz resulted in the expansion of the soft quartz due to the high vapor pressure in the ampoule, and often resulted in ampoule rupture. Bulk crystal growth of LiZnP requires temperatures slightly above the threshold of conventional resistive coil furnaces that typically have a $1200^{\circ} \mathrm{C}$ upper limit under continuous operation. Optical thermometer readings sampled through a quartz window of the crystal growth chamber, typically indicated between $1335-1355{ }^{\circ} \mathrm{C}$ (depending on the focus of the optical thermometer), which allowed for the congruent melt of LiZnP.

\section{II. $\quad X$-ray Diffraction Measurements}

The polished LiZnP sample is shown in Fig. 7. The sample was sandwiched between sheets of Mylar® for protection against moisture and air. The 4.077 x $3.518 \mathrm{~mm}$ sample face was evaluated for crystal structure, lattice constant, $d$ spacing, and orientation with a Bruker D2 CRYSO energy-dispersive x-ray diffractometer. The EDXRD method utilizes a Rhodium x-ray source that produces a polychromatic $\mathrm{x}$-ray beam, a monocapillary optic to collimate the $\mathrm{x}$-ray 
beam to a spot, and an energy dispersive silicon drift detector. Energy plots from the diffracted beam are collected as the sample is rotated about the crystal surface normal (phi). Peaks in the diffracted energy plots correspond to Laue reflections from the crystal. The out of plane $d$ spacing, degree of miscut and main orientation can be determined from these plots [26]. A collection of Laue peaks were observed for energy ranging between $5.0-6.5 \mathrm{keV}$, as shown in

186 Fig. 8. The mere observation of these peaks proved that the sample was crystalline. 187 Additionally, these peak locations were used to determine the main orientation, crystal structure, 188 lattice constant and $d$ spacing, by comparing to theoretical locations. A guess of the crystal structure, orientation and lattice constant was programed into the Bruker AXS Inc. software that calculated the theoretical peak positions based on the input parameters, and compared these locations to the experimental peak locations. The best fit to theoretical calculations was determined to be a cubic crystal structure with main orientation of (220), a lattice constant of $5.885 \AA$, and $d$ spacing of $2.081 \AA$. The lattice constant was slightly higher than that of what was determined from the synthesized material [1].

The expected phase identification pattern was determined for LiZnP using PowderCell 2.4, shown in Fig. 9 [27]. The calculation was based on the lattice constant determined from the EDXRD lattice constant result, $5.885 \AA$, and molybdenum optics of a $0.71 \AA$ wavelength. Highresolution phase identification scans were performed on the sample. Diffraction was observed at $2 \theta=19.4620^{\circ}$ and $\Omega=10.7294^{\circ}$. Next, a $\phi$ scan (sample is rotated along the azimuthal direction) was performed. From Fig. 10, it is evident that multiple facets are present. Intensity was maximized by adjusting the $\phi$ angle on the most intense peak i.e. $199.94^{\circ}$, and phase identification scans were collected as shown in Fig. 11. The (220) orientation was clearly prominent at a $2 \theta$ of $19.46^{\circ}$, which confirms with the EDXRD result. Additionally, the (440) orientation was observed at a $2 \theta$ of $39.48^{\circ}$ and matches well with the theoretical position. The (440) orientation was also seen in the EDXRD scan where corresponding Laue peaks were observed between $10.75-12.5 \mathrm{keV}$ as seen in Fig. 8. The crystalline ordering along the out of plane direction was examined by carrying out open detector rocking curves using a 4 bounce monochromator and an open detector. A (220) full width at half maximum (FWHM) of $0.417^{\circ}$ was recorded. A rocking curve performed on a Single Crystal Silicon standard (from Bruker AXS) yielded a FWHM of 0.004 degrees (14.4 arc seconds) for the Si (111) reflection, which indicates that the rocking curve FWHM broadening of the LiZnP sample is primarily coming from the mosaicity of the sample.

The $\psi$ axis (sample tilt) of the cradle, was utilized to explore the in-plane ordering of the sample. The angle between the (220) plane and other planes of interest are listed in Table 1. Diffraction was observed at a $\psi$ of $32.8560^{\circ}$. A phase identification scan was collected which produced three prominent orientations, which is further indication that the sample is not a perfect single crystal as shown in Fig. 13. The peak located at $22.6^{\circ} 2 \theta$ likely corresponds to the (311) orientation, which is theoretically located at $23.05^{\circ} 2 \theta$ for $\mathrm{LiZnP}$ with a lattice constant of 5.885 $\AA$. The peak located at $36.2^{\circ}$ matches nicely to the theoretical position of the (333) LiZnP with a lattice constant of $5.885 \AA$. However, the peak located at $26.3^{\circ} 2 \theta$ does not match well with the theoretical position for (222) LiZnP, with a lattice constant of $5.885 \AA$. These results indicate that grain boundaries exist in the evaluated area of the sample, and multiple domains could exist. Additionally, these domains may have a shift in lattice constant from what was seen in the (220) main orientation. At the same angle of $\psi\left(32.8560^{\circ}\right)$, the $2 \theta$ axis was positioned at the (311) reflection and a rocking curve was collected, as shown in Fig. 14. The FWHM was determined to be $0.294^{\circ}$. The same order of magnitude FWHM was determined for the out-of-plane (220) 
and off axis (311) measurements, indicating a reasonable degree of crystallinity in the out-of plane and in-plane directions.

\section{CONCLUSIONS}

The largest known crystalline ingots of LiZnP were grown by a modified Bridgman high

Table 1 . The angle between the (220) plane and the selected crystalline plane. temperature method. LiZnP crystal growth was performed successfully in pyrolytic graphite crucible lined tantalum vessels. The melting temperature observed was experimentally determined to be between $1320-1370^{\circ} \mathrm{C}$. A LiZnP sample exhibited a (220) main orientation with a lattice constant of $5.885 \AA$ as characterized by EDXRD and high-resolution XRD. A reasonable degree of crystallinity and ordering was observed for the out-of-plane and in-plane directions. Future work will include electrical characterization (resistivity, mobility-lifetime, trapping time), and neutron sensitivity measurements.

\begin{tabular}{cc}
\hline$(\boldsymbol{h} \boldsymbol{k l})$ plane & Angle \\
\hline$(422)$ & $30^{\circ}$
\end{tabular}
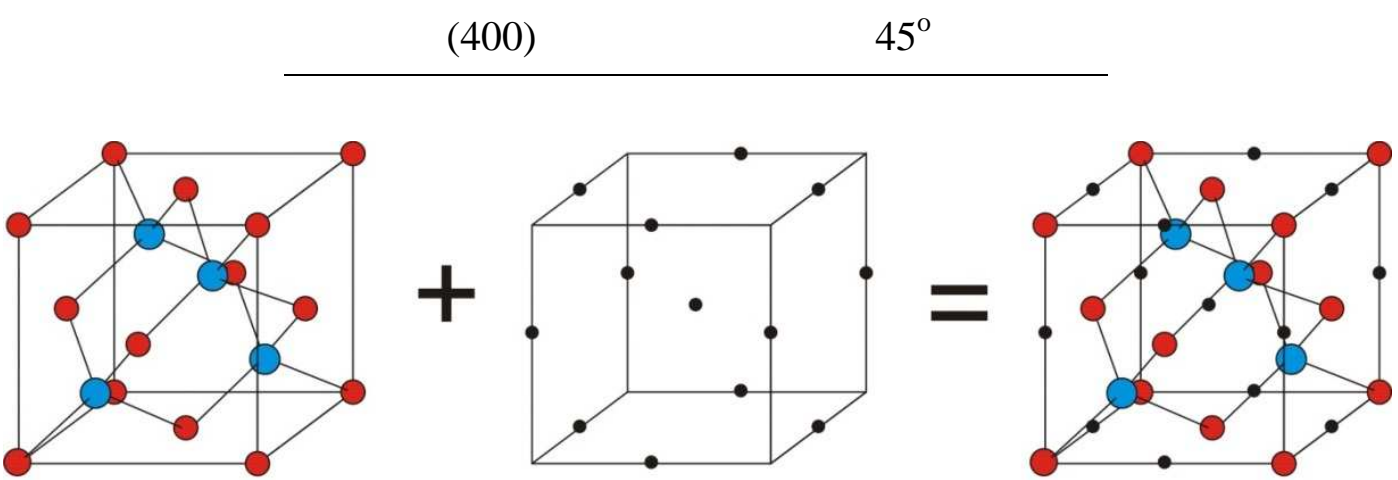

- Li $\odot$ Group II Group V

Fig. 1. The cubic crystal structure of $\mathrm{A}^{\mathrm{l}} \mathrm{B}^{\mathrm{II}} \mathrm{C}^{\mathrm{V}}$ compounds. 


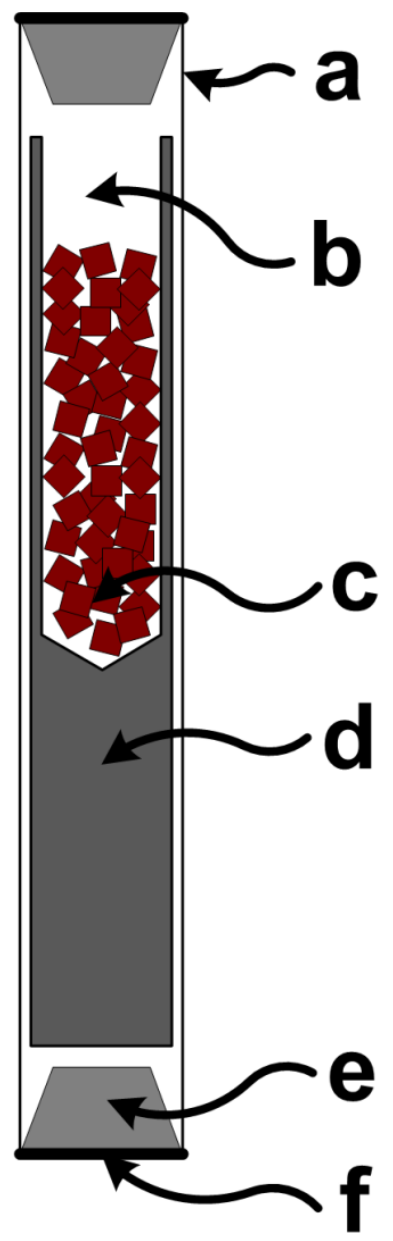

Fig. 2. Cross section of the loaded tantalum vessel. $\mathbf{a}-$ tantalum tube, $\mathbf{b}-$ argon gas, $\mathbf{c}-\mathrm{LiZnP}$ material, $\mathbf{d}$ - pyrolytic graphite crucible, $\mathbf{e}$ - tantalum cap, $\mathbf{f}$ - air-tight arc weld. 


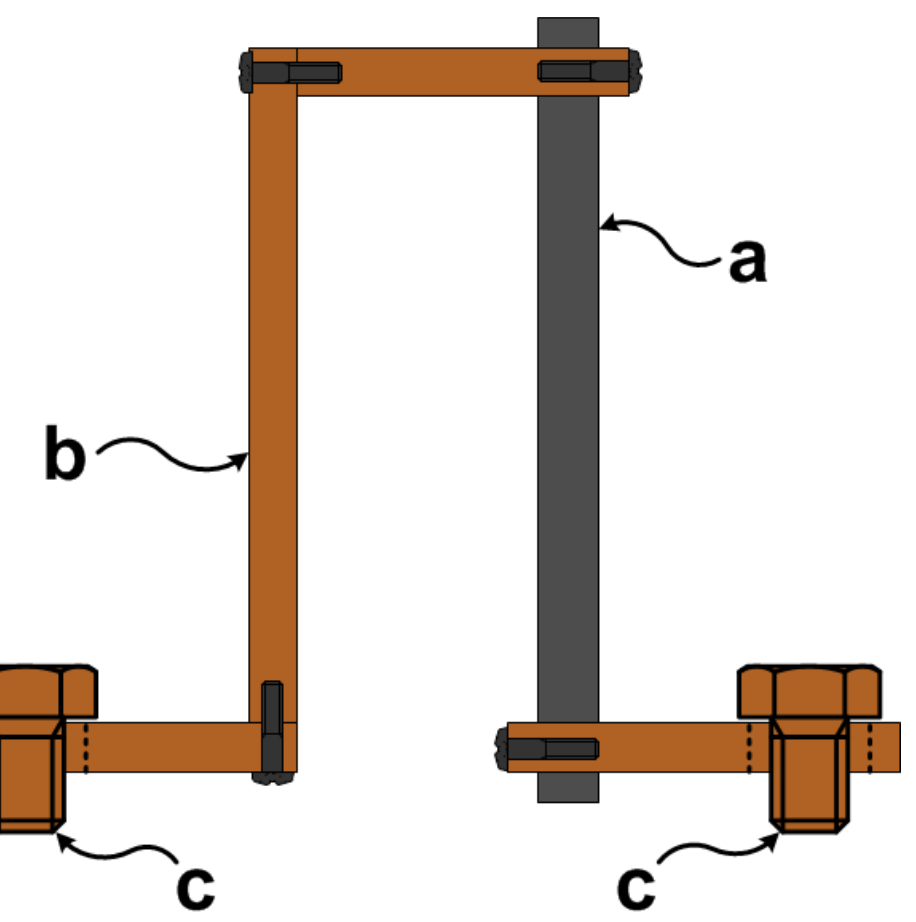

Fig. 3. Schematic of the oxygen-free copper harness. $\mathbf{a}-$ tantalum ampoule, $\mathbf{b}$ - oxygen-free copper, $\mathbf{c}$ - electrodes that connect to the low voltage power source.

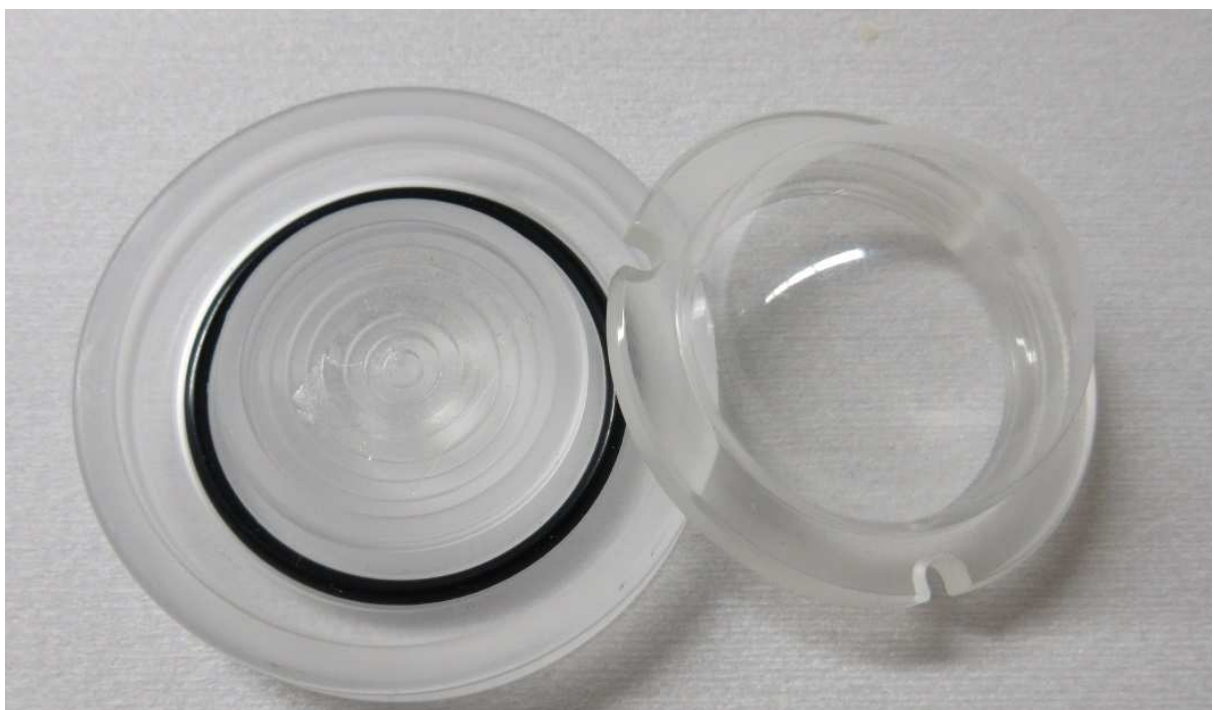
PMMA base with double-sided sticky tape (left) and PMMA dome (right) that screws firmly to the o-ring seal on the base. 
271

274

\section{7)}

Fig. 5. LiZnP crystals grown by the high-temperature growth method (picture collected through the glove box window).

\section{แด}

Fig. 6. Slice from the LiZnP purified ingot (picture collected through the glove box window).

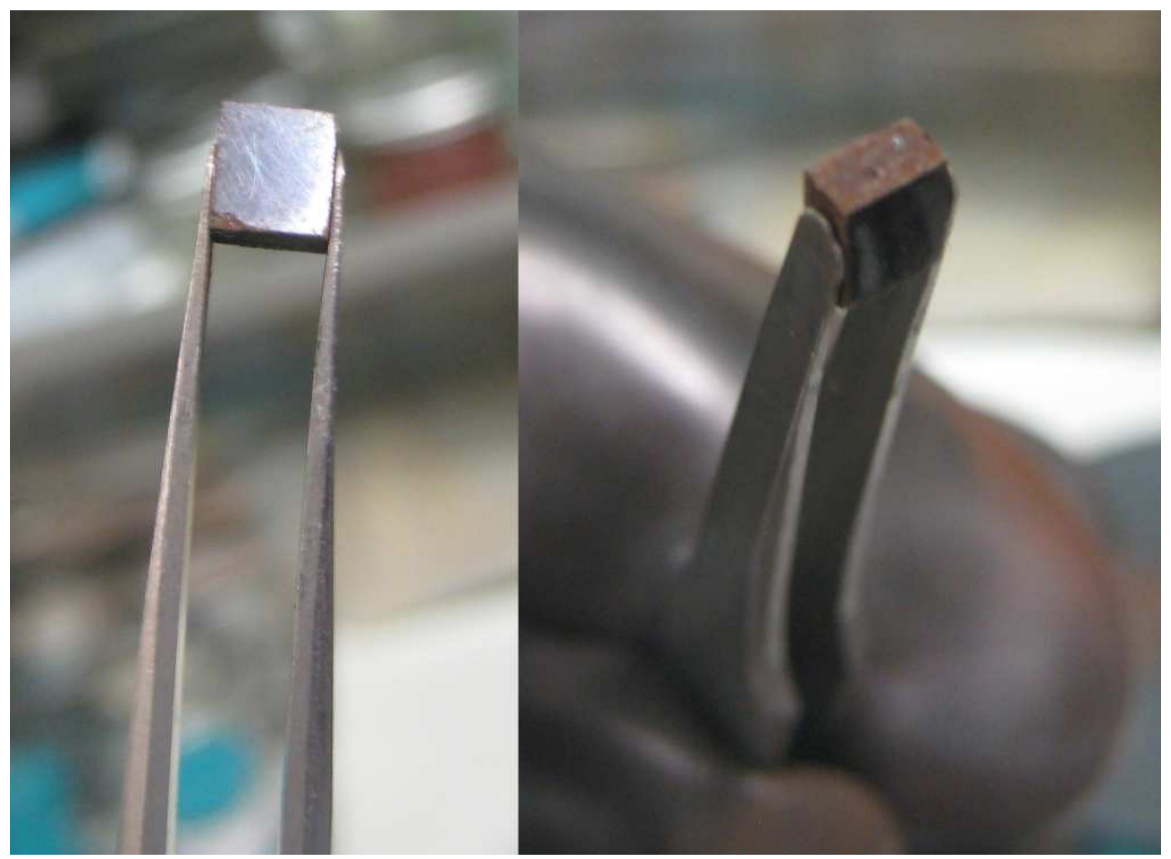

Fig. 7. A 4.077 x 3.518 x $2.020 \mathrm{~mm}$ purified bulk grown LiZnP sample 


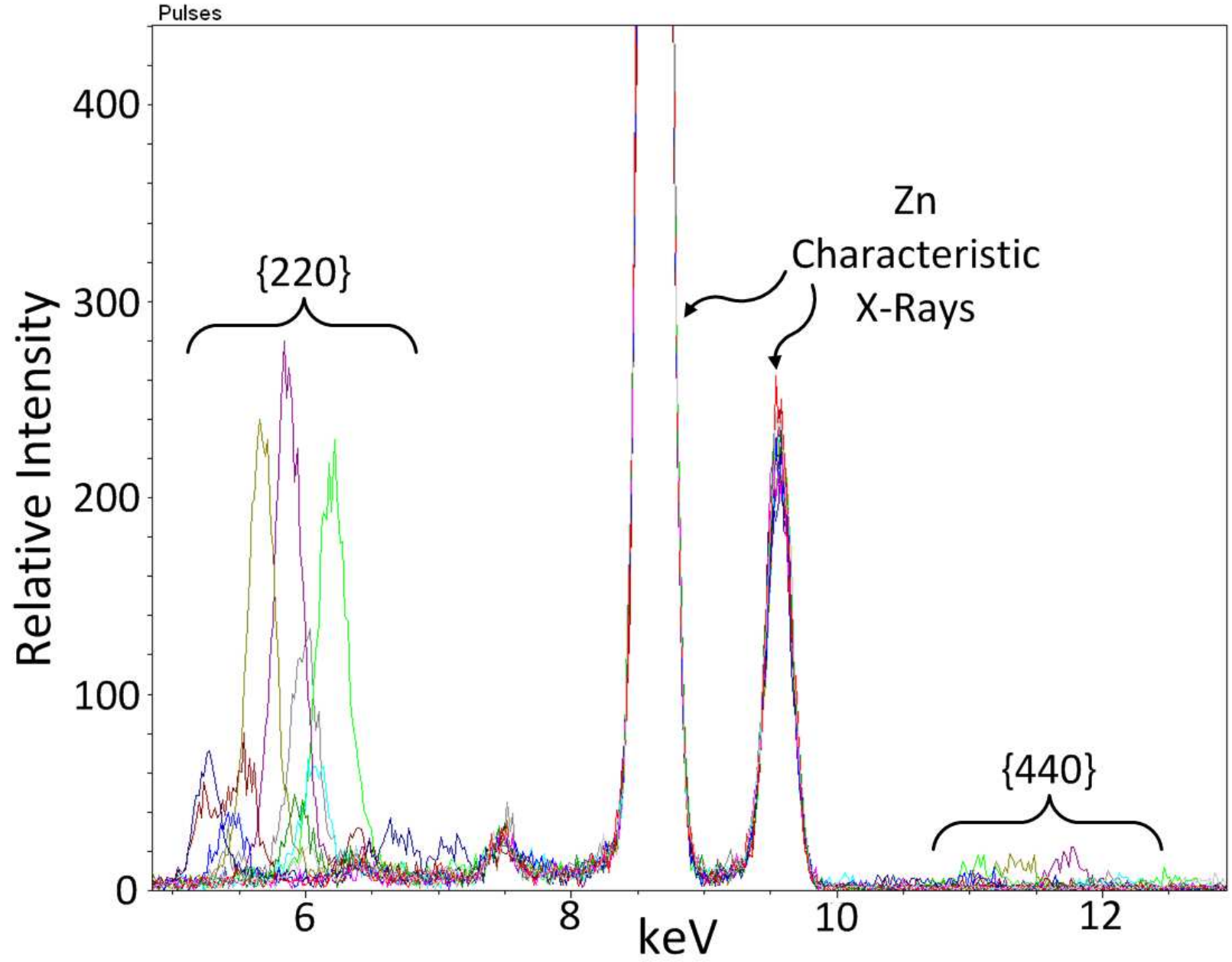

Fig. 8. The energy dispersive XRD spectrum collected on the polished LiZnP sample. Laue peaks from $\{220\}$ and the $\{440\} \mathrm{LiZnP}$ were observed. 


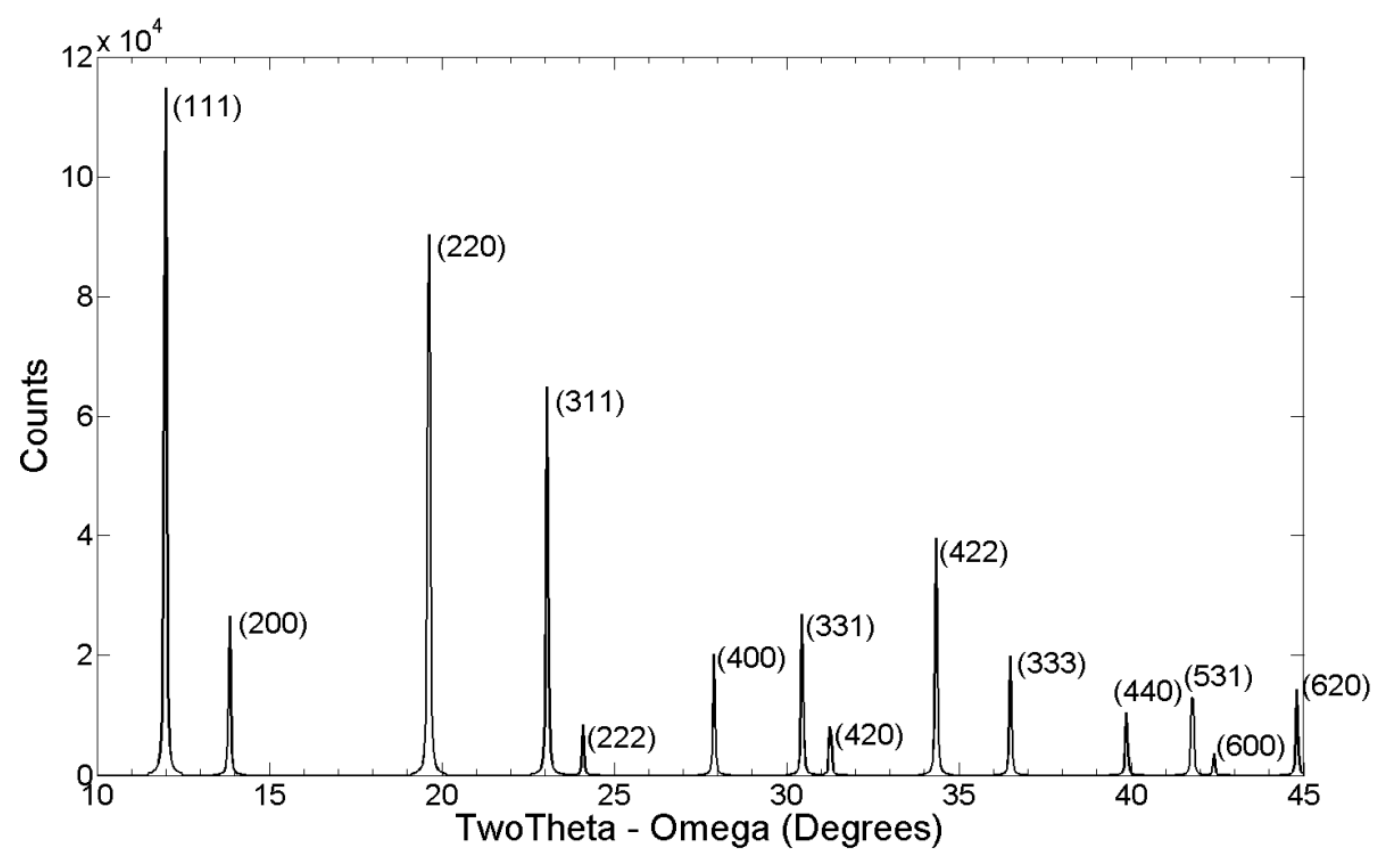

288 Fig. 9. The theoretical phase identification plot calculated with PowderCell 2.4 with a lattice 289 constant of $5.885 \AA$.

290

291

292

293

294

295

296

297

298

299

300

301

302

303

304

305

306

307 


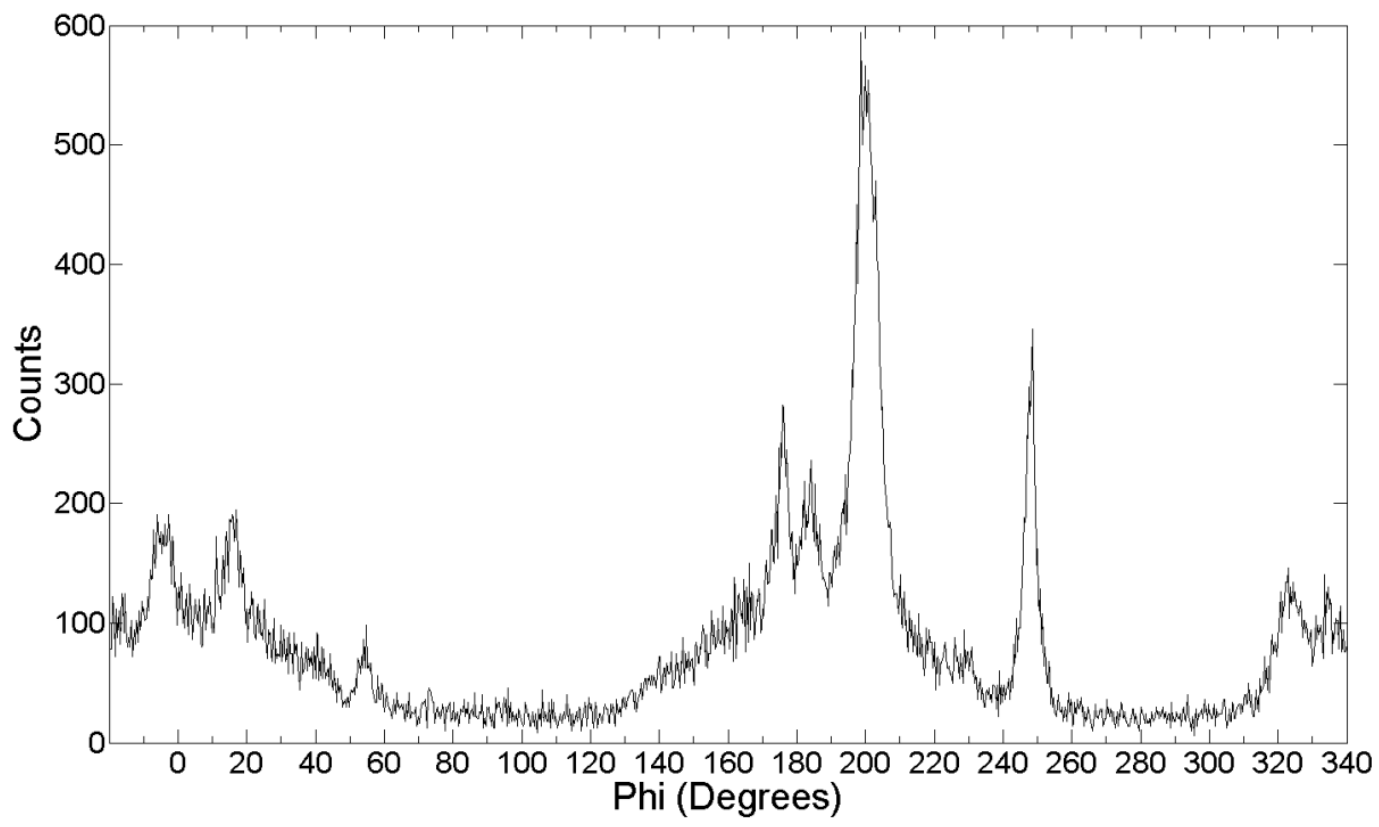

308

309

310

311

312

313

314

315
Fig. 10. A $\phi$ scan of the (220) main orientation. The array of peaks indicates presence multiple facets.

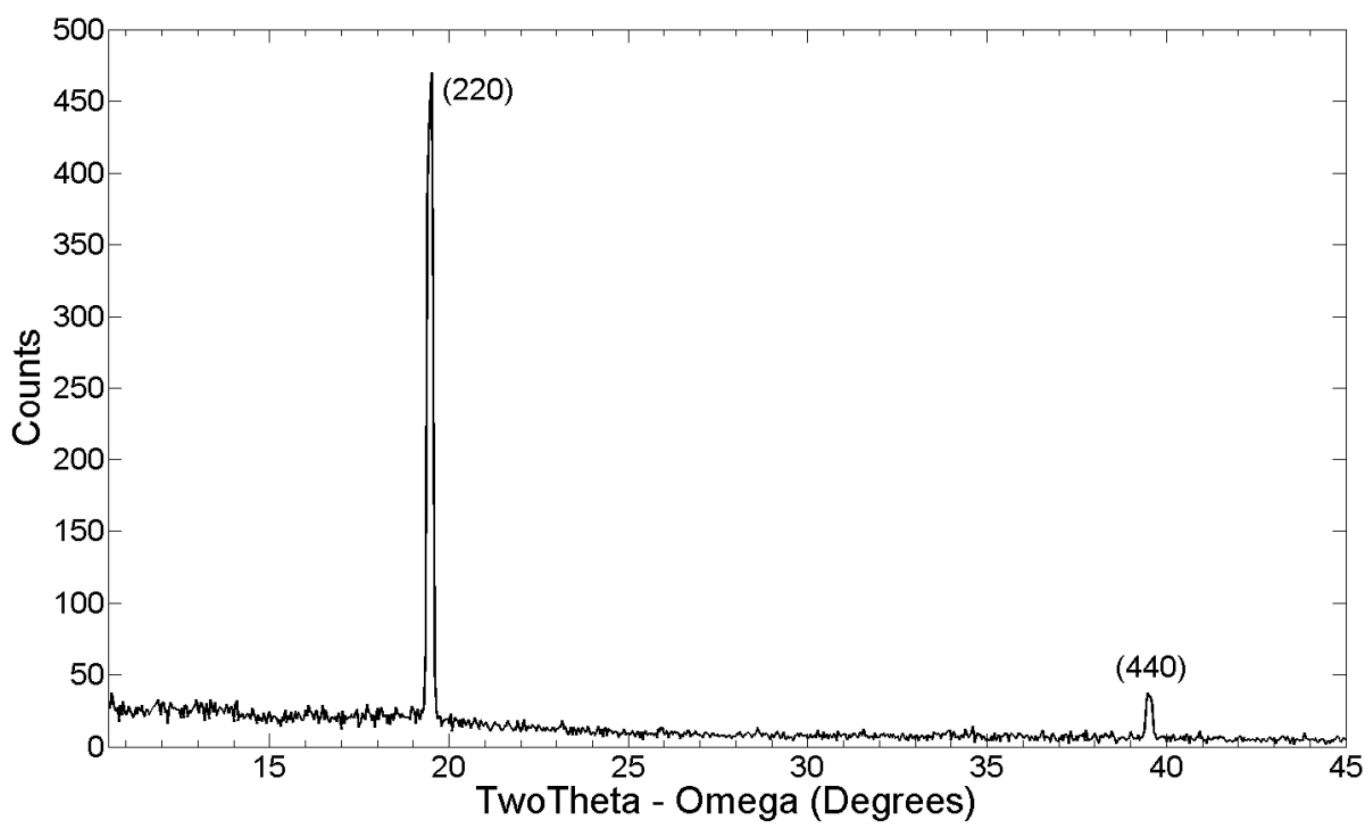

Fig. 11. Out-of-plane LiZnP phase identification scan. 


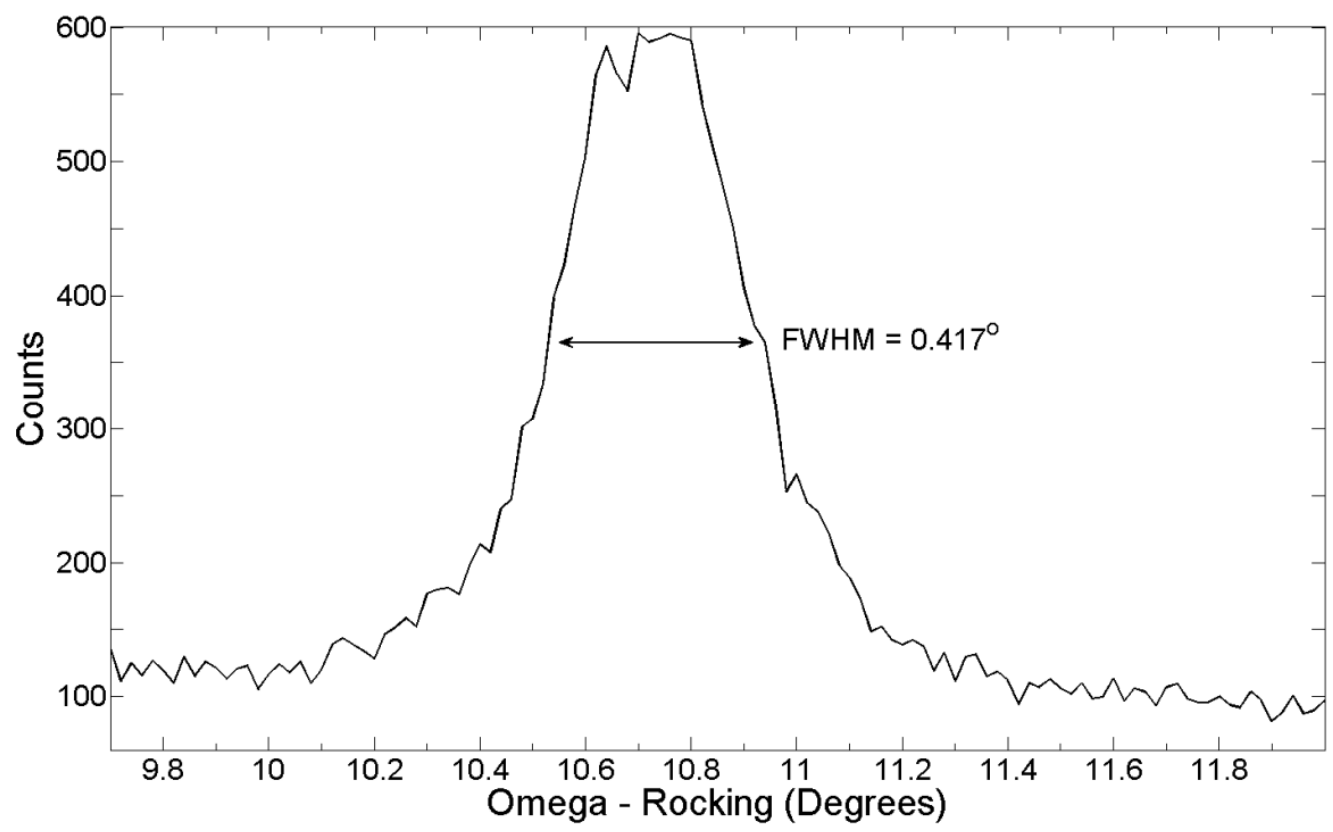

316

317

318

319
Fig. 12. Open detector (220) LiZnP rocking curve. A FWHM of $0.417^{\circ}$ was determined.

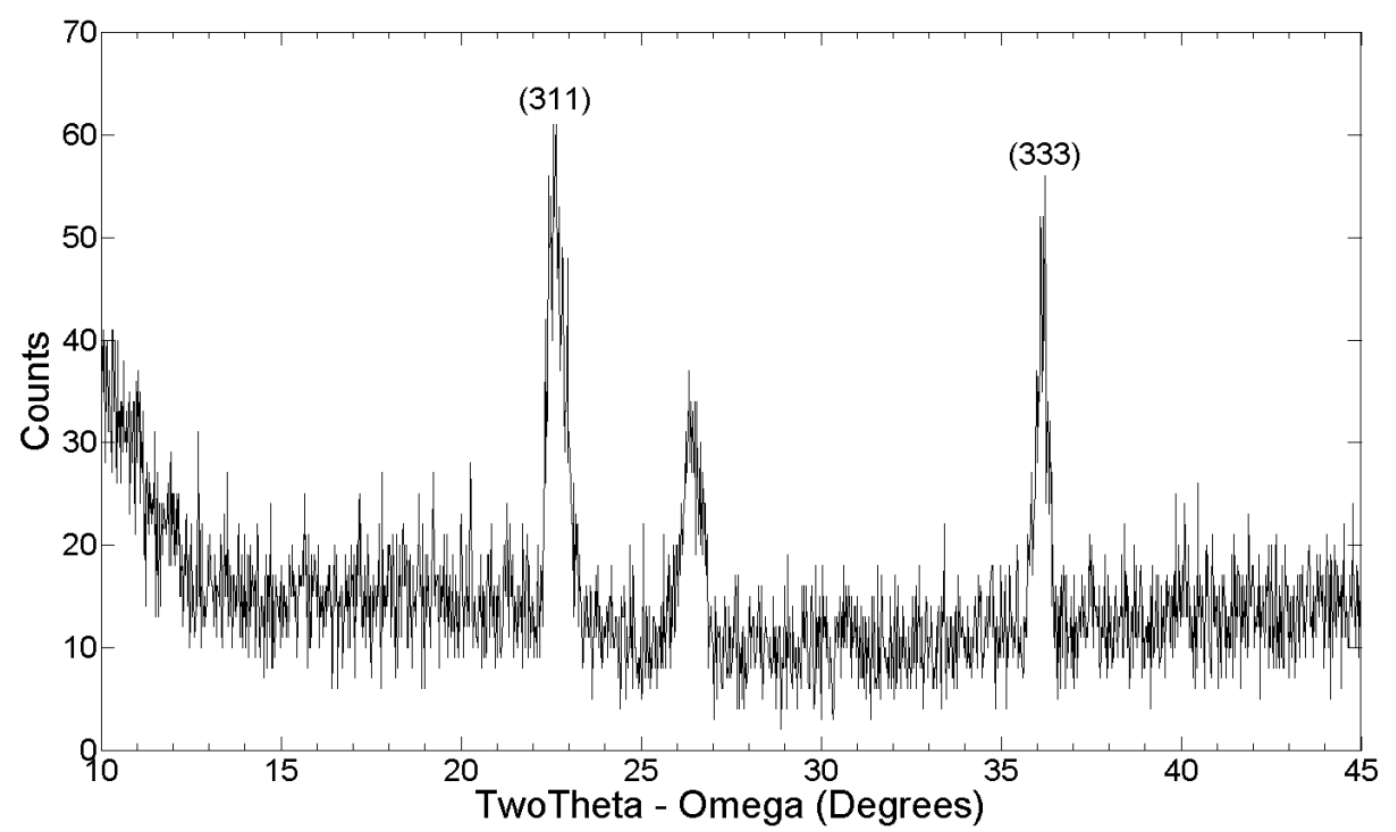

Fig. 13. A phase identification measurement at a $\psi$ of $32.8560^{\circ}$. The (311) and (333) orientations were identified. 


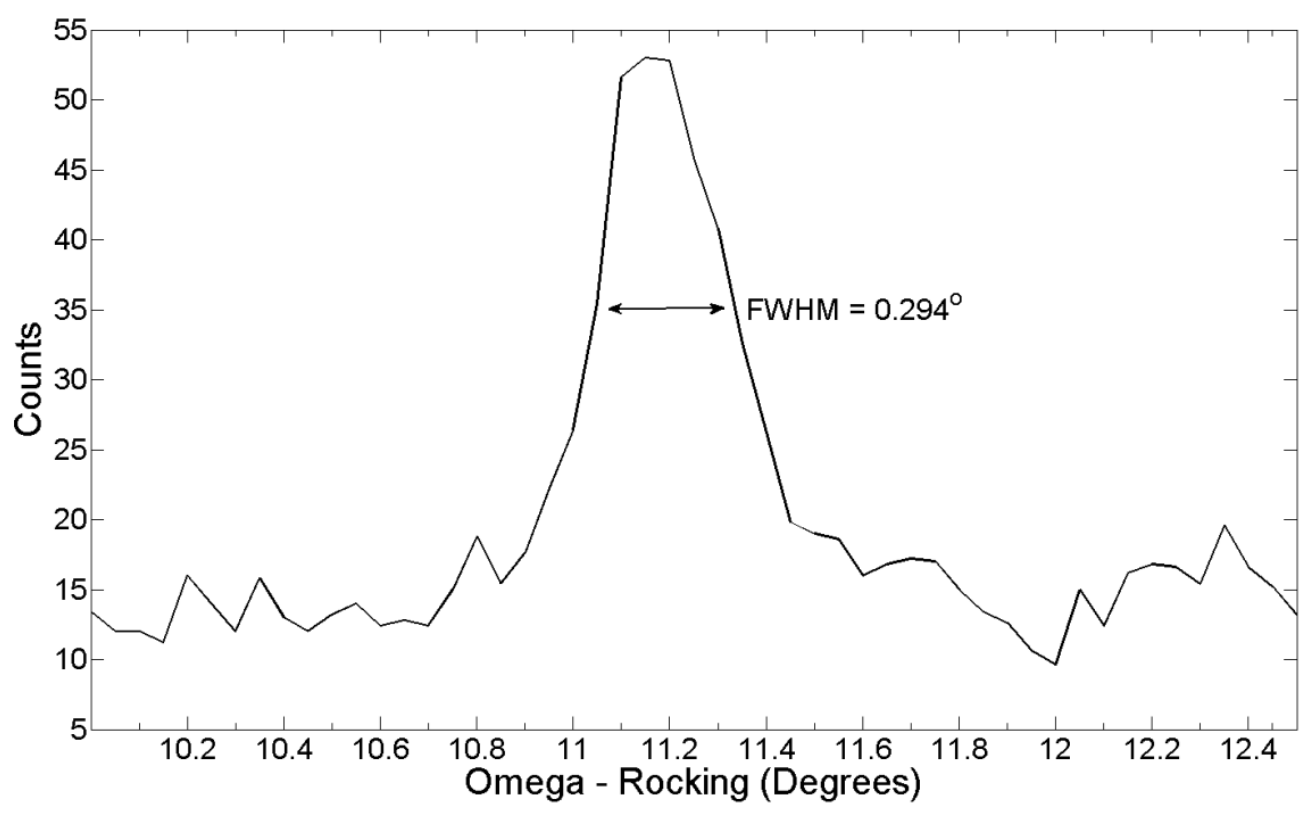

Fig. 14. Open detector (311) LiZnP rocking curve. A FWHM of $0.294^{\circ}$ was observed.

\section{ACKNOWLEDGEMENTS}

Work funded in part by the Advanced Materials program DOE - NNSA, grant \#DE-FG52-

\section{REFERENCES}

[1] B. W. Montag, M. A. Reichenberger, K. R. Arpin et al., "Synthesis and Characterization of LiZnP and LiZnAs Semiconductor Material," J. of Cryst. Growth, In Press, 2014.

[2] B. W. Montag, M. A. Reichenberger, P. B. Ugorowski et al., "Static Sublimation Purification Process and Characterization of LiZnP Semiconductor Material," J. of Cryst. Growth, Submitted, 2014.

[3] R. Juza, K. Langer, and K. Von Benda, "Ternary Nitrides, Phosphides, and Arsenides of Lithium," Angewandte Chemie International Edition in English, vol. 7, no. 5, pp. 360370, 1968.

[4] H. Nowotny, and K. Bachmayer, "Die Verbindungen LiMgP, LiZnP und LiZnAs," Monatshefte für Chemie und verwandte Teile anderer Wissenschaften, vol. 81, no. 4, pp. 488-496, 1950.

[5] D. Kieven, A. Grimm, A. Beleanu et al., "Preparation and properties of radio-frequencysputtered half-Heusler films for use in solar cells," Thin Solid Films, vol. 519, no. 6, pp. 1866-1871, 2011.

[6] K. Kuriyama, T. Katoh, and N. Mineo, "Crystal growth and characterization of the filled tetrahedral semiconductor lithium zinc phosphide (LiZnP)," J. Cryst. Growth, vol. 108, no. 1-2, pp. 37-40, 1991. 
[7] K. Kuriyama, and T. Katoh, "Optical band gap of the filled tetrahedral semiconductor lithium zinc phosphide (LiZnP)," Phys. Rev. B: Condens. Matter, vol. 37, no. 12, pp. 7140-2, 1988.

[8] R. Bacewicz, and T. F. Ciszek, "Preparation and characterization of some $\mathrm{A}^{\mathrm{I}} \mathrm{B}^{\mathrm{II}} \mathrm{C}^{\mathrm{V}}$ type semiconductors," Appl. Phys. Lett., vol. 52, no. 14, pp. 1150-1, 1988.

[9] Z. W. Bell, K. R. Pohl, and L. Van Den Berg, "Neutron detection with mercuric iodide," IEEE Trans. Nuc. Sci., vol. 51, no. 3, pp. 1163-1165, 2004.

[10] A. G. Vradii, M. I. Krapivin, L. V. Maslova et al., "Possibilities of recording thermal neutrons with cadmium telluride detectors," Soviet Atomic Energy, vol. 42, no. 1, pp. 6466, 1977.

[11] A. G. Beyerle, and K. L. Hull, "Neutron detection with mercuric iodide detectors," Nucl. Inst. and Meth. A, vol. 256, no. 2, pp. 377-380, 1987.

[12] D. S. McGregor, J. T. Lindsay, and R. W. Olsen, "Thermal neutron detection with cadmium $_{1-\mathrm{x}}$ zinc $_{\mathrm{x}}$ telluride semiconductor detectors," Nucl. Inst. and Meth. A, vol. 381, no. 2, pp. 498-501, 1996.

[13] Y. Kumashiro, K. Kudo, K. Matsumoto et al., "Thermal neutron irradiation experiments on ${ }^{10} \mathrm{BP}$ single-crystal wafers," Journal of the Less Common Metals, vol. 143, no. 1-2, pp. 71-75, 1988.

[14] W. C. McGinnis, "Film Implementation of a Neutron Detector (FIND): Proof of Concept Device," Technical Report 1921, 2003.

[15] J. C. Lund, F. Olschner, F. Ahmed et al., "Boron Phosphide on Silicon for Radiation Detectors," MRS Online Proceedings Library, vol. 162, pp. 601-604, 1989.

[16] F. P. Doty, Boron Nitride Solid State Detector, US-6, 504, April 27, 2004, US-6,727,504, April 27, 2004.

[17] A. N. Caruso, P. A. Dowben, S. Balkir et al., "The all boron carbide diode neutron detector: Comparison with theory," Mater. Sci.and Eng. B, vol. 135, no. 2, pp. 129-133, 2006.

[18] B. W. Robertson, S. Adenwalla, A. Harken et al., "A class of boron-rich solid-state neutron detectors," Appl. Phy. Let., vol. 80, no. 19, pp. 3644-3646, 2002.

[19] P. Groot, J. H. F. Grondel, and P. J. v. d. Put, "Chemical Vapour Deposition of Boron Phosphides Using Bromide Etchants," Solid State Ionics, vol. 16, pp. 95-98, 1985.

[20] A. N. Caruso, R. B. Billa, S. Balaz et al., "The Heteroisomeric Diode," J. Phys. Condens. Matter, vol. 16, pp. L139-L146, 2004.

[21] D. S. McGregor, M. D. Hammig, Y. H. Yang et al., "Design considerations for thin film coated semiconductor thermal neutron detectors-I: basics regarding alpha particle emitting neutron reactive films," Nucl. Inst. and Meth. A, vol. 500, no. 1-3, pp. 272-308, 2003.

[22] R. Bacewicz, and T. F. Ciszek, "Crystal growth and characterization of some novel $\mathrm{A}^{\mathrm{I}} \mathrm{B}^{\mathrm{II}} \mathrm{C}^{\mathrm{V}}$ semiconductors," Acta Phys. Pol., A, vol. A77, no. 2-3, pp. 379-81, 1990.

[23] K. Kuriyama, T. Katoh, and S. Tsuji, "Preparation and characterization of the filled tetrahedral semiconductor lithium zinc phosphide film on quartz," J. Appl. Phys., vol. 66, no. 8, pp. 3945-7, 1989.

[24] K. Geels, Metallographic and Materialographic Specimen Preparation, Light Microscopy, Image Analysis and Hardness Testing, West Conshohocken, PA 194282959: ASTM International, 2007. 
[25] Pace Technologies, "Abrasive Grinding Paper,"

[26] Bruker AXS Inc, "brochure_D2_CRYSO.pdf,"

399

400

401

402

403

http://bruker.poznan.pl/images/stories/AXS/brochure_D2_CRYSO.pdf, 2009.

404

[27] W. Kraus, and G. Nolze, "POWDER CELL - a program for the representation and manipulation of crystal structures and calculation of the resulting X-ray powder patterns," J. Appl. Crys., vol. 29, no. 3, pp. 301-303, 1996. 
Table(s)

\begin{tabular}{cc}
\hline$(\boldsymbol{h} \boldsymbol{k} \boldsymbol{l})$ plane & Angle \\
\hline$(422)$ & $30^{\circ}$ \\
$(311)$ & $31.48^{\circ}$ \\
$(222)$ & $35.26^{\circ}$ \\
$(400)$ & $45^{\circ}$ \\
\hline
\end{tabular}

